\title{
Manipulation of thin silver film growth on weakly interacting silicon dioxide substrates using oxygen as a surfactant
}

Nikolaos Pliatsikas, Andreas Jamnig, Martin Konpan, Andreas Delimitis, Gregory Abadias and Kostas Sarakinos

The self-archived postprint version of this journal article is available at Linköping University Institutional Repository (DiVA):

http://urn.kb.se/resolve?urn=urn:nbn:se:liu:diva-167283

N.B.: When citing this work, cite the original publication.

Pliatsikas, N., Jamnig, A., Konpan, M., Delimitis, A., Abadias, G., Sarakinos, K., (2020), Manipulation of thin silver film growth on weakly interacting silicon dioxide substrates using oxygen as a surfactant, Journal of Vacuum Science \& Technology. A. Vacuum, Surfaces, and Films, 38(4), 043406.

https://doi.org/10.1116/6.0000244

Original publication available at:

https://doi.org/10.1116/6.0000244

Copyright: American Vacuum Society

http://www.avs.org/ 


\section{Manipulation of thin silver film growth on weakly-interacting silicon dioxide substrates using oxygen as a surfactant}

Running title: Manipulation of thin silver film growth on weakly-interacting silicon dioxide substrates using oxygen as a surfactant

Running Authors: Nikolaos Pliatsikas et al.

Nikolaos Pliatsikas ${ }^{1}$, Andreas Jamnig ${ }^{1,2}$, Martin Konpan ${ }^{1}$, Andreas Delimitis ${ }^{3}$, Gregory Abadias ${ }^{2}$, and Kostas Sarakinos ${ }^{1, a)}$

${ }^{1}$ Nanoscale Engineering Division, Department of Physics, Chemistry and Biology, Linköping University, Linköping, SE 581 83, Sweden

${ }^{2}$ Institut Pprime, Département Physique et Mécanique des Matériaux, UPR 3346 CNRS, Université de Poitiers, SP2MI, 11 Bvd M. et P. Curie, F86073 Poitiers Cedex 9, France

${ }^{3}$ Department of Mechanical and Structural Engineering and Materials Science, University of

Stavanger, N-4036 Stavanger, Norway

a) Electronic mail: kostas.sarakinos@liu.se

We study the morphological evolution of magnetron-sputtered thin silver (Ag) films which are deposited on weakly-interacting silicon dioxide $\left(\mathrm{SiO}_{2}\right)$ substrates in an oxygencontaining $\left(\mathrm{O}_{2}\right)$ gas atmosphere. In situ and real-time monitoring of electrically-conductive layers, along with ex situ microstructural analyses, show that presence of $\mathrm{O}_{2}$, throughout all film-formation stages, leads to a more pronounced two-dimensional (2D) morphology, smoother film surfaces, and larger continuous-layer electrical resistivities, as compared to Ag films grown in pure argon (Ar) ambient. In addition, our data demonstrate that 2D morphology can be promoted, without compromising the Ag-layer electrical conductivity, if $\mathrm{O}_{2}$ is deployed with high temporal precision to target film formation stages before the formation of a percolated layer. Detailed real-space imaging of discontinuous films, augmented by in situ growth monitoring data, suggest that $\mathrm{O}_{2}$ favors $2 \mathrm{D}$ morphology by affecting the kinetics of initial film-formation stages, and most notably by decreasing the 
rate of island coalescence completion. Furthermore, compositional and bonding analyses show that $\mathrm{O}_{2}$ does not change the chemical nature of the Ag layers and no atomic oxygen is detected in the films, i.e., $\mathrm{O}_{2}$ acts as a surfactant. The overall results of this study are relevant for developing non-invasive surfactant-based strategies for manipulating noblemetal-layer growth on technologically relevant weakly-interacting substrates, including graphene and other 2D crystals.

\section{INTRODUCTION}

Growth of thin noble-metal films with two-dimensional (2D) morphology on weakly-interacting 2D-material and oxide substrates is a crucial step in the fabrication of multifunctional contacts in a wide array of key enabling devices ${ }^{1-7}$. Achieving such morphology, so that the metal-layer fully wets the underlying substrate, entails a great scientific challenge: the adsorption energy of noble-metal atoms on weakly-interacting surfaces is significantly smaller than the bulk-metal binding energy ${ }^{4,7}$, thereby providing the driving force toward a pronounced and uncontrolled three-dimensional (3D) growth.

Vapor-based film deposition is a far-from-equilibrium process in which morphology is predominantly determined by the kinetic rates of atomic-scale structureforming processes during early growth stages ${ }^{8-11}$. The kinetic pathways leading to $3 \mathrm{D}$ morphologies in homo- and hetero-epitaxial film/substrate systems are well established in the literature ${ }^{11}$. This understanding has enabled the development of growth manipulation strategies in which surfactants (i.e., minority metal or gaseous species) are deployed and temporary adsorbed at the film growth front to allow navigation between $2 \mathrm{D}$ and $3 \mathrm{D}$ morphologies ${ }^{12-26}$. 
The atomistic mechanisms that govern morphological evolution of metal films on weakly-interacting substrates are different from those in strongly-interacting epitaxial systems $^{7,27-29}$. As such, established knowledge for surfactant-based growth manipulation is not directly applicable to the case of noble-metal film deposition on 2D materials and oxides. Despite the latter, there are empirical studies in which less-noble-metal surfactants and seed layers ${ }^{30-35}$, as well as gaseous surfactants ${ }^{36-41}$ have been used to suppress the 3D morphology of silver and copper films on oxide substrates.

Recently, we have contributed to the fundamental understanding of the mechanisms that govern surfactant-modified film growth on weakly-interacting substrates by studying the effect of nitrogen $\left(\mathrm{N}_{2}\right)$ gas on the morphological evolution of silver $(\mathrm{Ag})$ films on silicon dioxide $\left(\mathrm{SiO}_{2}\right)^{42}$. Our results showed that $\mathrm{N}_{2}$ affects the various film-formation stages in a complex manner: when $\mathrm{N}_{2}$ is present during island nucleation and coalescence, 2D growth morphology is promoted; while the opposite is observed when $\mathrm{N}_{2}$ is deployed after island coalescence is completed, so that the stage of hole-filling is primarily affected.

The goal of the present work is to understand the role of chemical affinity between noble-metal and gas surfactant species on the various film-formation stages and the overall film morphological evolution on weakly-interacting substrates. To this purpose, we study the growth of magnetron-sputtered $\mathrm{Ag}$ films on $\mathrm{SiO}_{2}$ substrates, in an oxygen-containing $\left(\mathrm{O}_{2}\right)$ gas atmosphere. We use in situ and real-time spectroscopic ellipsometry to monitor the evolution of optoelectronic properties of electricallyconductive layers and, in combination with ex situ microstructural analysis, we establish 
that presence of $\mathrm{O}_{2}$ throughout all film-formation stages, leads to a more pronounced 2D morphology, smoother film surfaces, but larger continuous-layer electrical resistivities, relative to Ag films grown in a pure argon (Ar) atmosphere. These trends are qualitatively consistent with the effect of $\mathrm{N}_{2}$ on Ag-layer morphology ${ }^{42,43}$. However, a $\sim 10$ times smaller $\mathrm{O}_{2}$ partial pressure is required for the surfactant effect to manifest itself; which can be explained by the higher reactivity of $\mathrm{O}_{2}$ toward $\mathrm{Ag}$, as compared to that of $\mathrm{N}_{2}{ }^{44-46}$. In addition, our in situ and real-time data demonstrate that the detrimental effect of $\mathrm{O}_{2}$ on Ag-layer conductivity can be mitigated, while promoting 2D morphology, if $\mathrm{O}_{2}$ is deployed in a way that targets initial film-growth stages before the formation of a percolated layer. Detailed ex situ real-space imaging, combined with data from in situ and real-time monitoring of discontinuous metal-layer growth, suggest that $\mathrm{O}_{2}$ favors $2 \mathrm{D}$ morphology by affecting the kinetics of initial film-formation stages - most notably by decreasing the rate of island coalescence completion. Moreover, compositional and bonding analyses show that the presence of $\mathrm{O}_{2}$ in the gas atmosphere does not affect the chemical nature of the metal layer in which only Ag-Ag bonds are present, while no atomic oxygen is detected in the bulk of the film, thereby showing that $\mathrm{O}_{2}$ acts as a surfactant.

\section{EXPERIMENTAL STRATEGY AND PROCEDURES}

\section{A. Film synthesis}

Thin Ag films are synthesized by direct current magnetron sputtering (dcMS) at a constant current of $20 \mathrm{~mA}$, resulting in a deposition rate of $\sim 0.11 \mathrm{~nm} / \mathrm{s}$. All depositions

are carried out in a multi-source ultra-high vacuum chamber (based pressure $\sim 10^{-8} \mathrm{~Pa}$ ) on 
Czochralski-grown n-type $\operatorname{Si}(100)$ wafers, covered with a $\sim 530 \mathrm{~nm}$ thick thermallygrown $\mathrm{SiO}_{2}$ layer. No intentional substrate heating is used during the depositions. The magnetron source, equipped with a $\mathrm{Ag}$ target (diameter $7.62 \mathrm{~cm}$, purity $99.99 \%$ ), is placed $7.5 \mathrm{~cm}$ from the substrate, and at an angle of $45^{\circ}$ with respect to the substrate surface normal.

$\operatorname{Ar}$ (purity 99.999\%) is used as a sputtering gas. Films are deposited either in pure Ar atmosphere or in $\mathrm{Ar} / \mathrm{O}_{2}$ mixtures $\left(\mathrm{O}_{2}\right.$ purity $\left.99.999 \%\right)$ at a total working pressure $p_{\text {total }}$ of 1.3 Pa. For samples grown in $\mathrm{Ar} / \mathrm{O}_{2}$ atmospheres, initial tests at different $\mathrm{O}_{2}$ partial pressures $\mathrm{p}_{\mathrm{O}_{2}}$ showed no appreciable difference in the film morphological evolution for $\frac{p_{\mathrm{O}_{2}}}{p_{\text {total }}}>0.01$. Hence, in the remainder of the article we focus on experiments performed for $p_{O_{2}}=0.01 \times p_{\text {total }}$. It should be pointed out that the partial pressures refer to values measured in the absence of plasma. Moreover, the Ar introduction point is at the chamber wall halfway between the substrate holder and the magnetrons, while $\mathrm{O}_{2}$ is introduced through an orifice in the vicinity of the substrate. A schematic illustration of the chamber layout, including the spectroscopic ellipsometer used for in situ film growth monitoring (see section II.B) and the gas introduction points is shown in Fig. 1. 


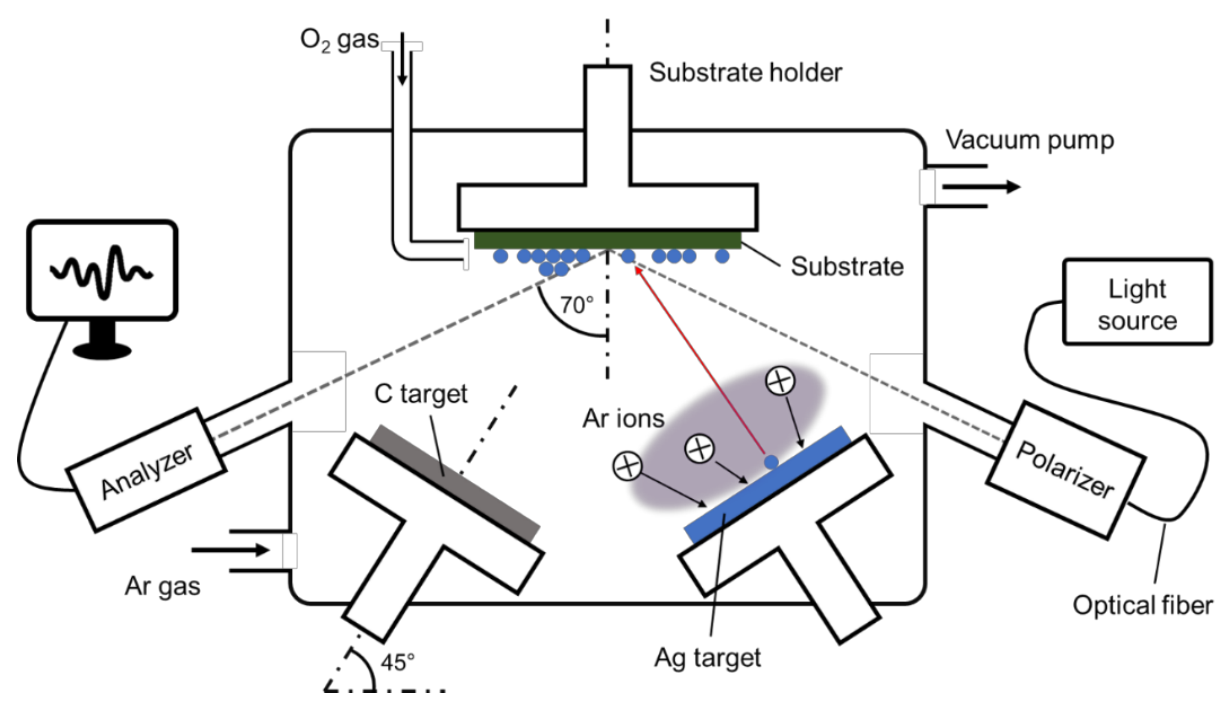

FIG. 1. Schematic illustration of the deposition chamber, including the $\mathrm{Ar}$ and $\mathrm{O}_{2}$ gas introduction points and the spectroscopic ellipsometer used for in situ film growth monitoring.

We have recently studied ${ }^{42}$ the growth of magnetron sputter-deposited Ag layers on $\mathrm{SiO}_{2}$ in mixed $\mathrm{Ar} / \mathrm{N}_{2}$ gas atmospheres, and we established that $\mathrm{N}_{2}$ affects the various film-formation stages in distinctly different ways. In order to explore whether the latter is also the case when $\mathrm{O}_{2}$ is used to manipulate growth, we deploy $\mathrm{O}_{2}$ using the following schemes: (i) $\mathrm{O}_{2}$ is present together with Ar throughout the entire deposition process; (ii) $\mathrm{O}_{2}$ is introduced at the early film-growth stages so that the growth surface is exposed to the presence of surfactant for a time $t_{E}$ ranging between 2 to $20 \mathrm{~s}$. Subsequently, $\mathrm{O}_{2}$ is pumped out from the deposition chamber, while deposition continues and is completed in pure Ar atmosphere; and (iii) deposition commences in a pure Ar ambient and, after a delay time $t_{D}$ ranging from 2 to $20 \mathrm{~s}, \mathrm{O}_{2}$ is introduced for the remainder of the growth.

The film morphological evolution, microstructure, and chemical composition are analyzed using the in situ and ex situ tools and methodologies described in section II.B. 
Prior to exposing the samples to atmosphere for ex situ analyses, and immediately after Ag deposition, samples are capped with a 3 nm-thick amorphous carbon (a-C) layer to avoid surface contamination and changes in film morphology upon atmospheric exposure. The a-C capping layers are sputter-deposited at a rate of $0.01 \mathrm{~nm} / \mathrm{s}$ from a graphite (C) target (purity 99.99 at.\%; diameter $7.6 \mathrm{~cm}$; thickness $6 \mathrm{~mm}$ ) in pure Ar at 1.3 $\mathrm{Pa}$. The $\mathrm{C}$ target is operated in dcMS at a constant current mode of $50 \mathrm{~mA}, 7.5 \mathrm{~cm}$ away from the substrate, and at an angle of $45^{\circ}$ with the substrate surface normal.

\section{B. In situ film growth monitoring and ex situ film characterization}

Spectroscopic ellipsometry is employed in situ and in real-time to monitor the change of the film optoelectronic properties during deposition and, thereby, draw conclusions about the film morphological evolution. Data are collected every $\sim 2 \mathrm{~s}$ in the range 1.6-3.2 eV, at an angle of incidence of $\sim 70^{\circ}$ from the substrate normal, using a rotating analyzer ellipsometer. The acquired data are fitted to a three-phase model consisting of vacuum, metal-layer, and substrate. The substrate optical properties are described by a semi-infinite $625 \mu \mathrm{m} \mathrm{Si}$ slab with a top layer of $\mathrm{SiO}_{2}$, the thickness of which is determined by measuring the optical response of the substrate prior to deposition. Optical constants for the $\mathrm{Si}$ and $\mathrm{SiO}_{2}$ layers are taken from Ref. ${ }^{47}$ and Ref. ${ }^{48}$. The optical response of the film is described using the Drude-Lorentz dispersion model, as detailed below.

During initial growth stages, the film surface primarily features isolated $\mathrm{Ag}$ islands that give rise to localized surface plasmon resonance (LSPR) ${ }^{49,50}$, which can be 
described by adapting the Lorentz oscillator model ${ }^{49,50}$ to express the complex dielectric function of the layer $\tilde{\epsilon}(\omega)$ as

$$
\tilde{\epsilon}(\omega)=\frac{f \omega^{2}}{\omega_{0}^{2}-\omega^{2}-i \Gamma \omega}
$$

In Eq. (1), $f$ and $\omega_{o}$ are the oscillator strength and resonance frequency, respectively, and $\Gamma$ represents the damping rate of the plasmon resonance. More details on the implementation and relevance of the Lorentz oscillator model for describing the optical response of discontinuous $\mathrm{Ag}$ layers can be found in our previous work ${ }^{42}$.

The optical response of electrically-conductive Ag films is described by the Drude free electron theory ${ }^{51}$, according to which the dielectric function $\tilde{\epsilon}_{D}(\omega)$ is given by the expression,

$$
\tilde{\epsilon}_{D}(\omega)=\epsilon_{\infty}-\frac{\omega_{p}^{2}}{\omega^{2}+i \Gamma_{D} \omega}
$$

In Eq. (2), $\epsilon_{\infty}$ is a constant that accounts for the effect of interband transitions occurring at frequencies higher than the ones considered here, $\Gamma_{D}$ is the free-electron damping constant, and $\omega_{p}=\sqrt{n e^{2} / \varepsilon_{0} m_{e}}$ is the free-electron plasma energy, where $n$ is the freeelectron density, $e$ is the electric charge, $m_{e}$ is the free-electron effective mass, and $\varepsilon_{0}$ is the permittivity of free space. From Eq. (2), the room-temperature film resistivity is calculated as ${ }^{51}$

$$
\rho=\frac{\Gamma_{D}}{\epsilon_{0} \omega_{p}^{2}}
$$

Besides the optical properties, the analysis of the ellipsometric data enable us to calculate the film height $h_{f}$ as a function of deposition time $t$. Using the continuous-layer 
$h_{f}$ value, the film deposition rate $F$ is extracted $(F \approx 0.11 \mathrm{~nm} / s$ at all conditions used in this work), from which the nominal film thickness $\Theta$ (i.e., the amount of deposited material) at any given time during growth $t$ is calculated as $\Theta=F \times t$. Throughout the manuscript, $\Theta$ is expressed in monolayers (ML), whereby one ML corresponds to the amount of atoms per unit area that is required to increase the film thickness by an amount equal to the $\mathrm{Ag}(111)$ interplanar spacing $(0.235 \mathrm{~nm})$, which is the most common out-ofplane growth orientation for physical vapor deposited face-centered-cubic (fcc) metal films.

In situ characterization is complemented by ex situ imaging of discontinuous film surfaces using a Field Emission Gun Scanning Electron Microscope (SEM), at an operating voltage of $4 \mathrm{kV}$ and a working distance of $3 \mathrm{~mm}$. SEM images are analyzed using the ImageJ software package ${ }^{52}$, to determine the fraction of the substrate covered by the film, as well as the island size and shape distribution.

Real-space imaging is combined with $\mathrm{x}$-ray reflectometry (XRR) to obtain film roughness $w$, thickness $h_{f}$, and mass density $\rho_{m}$. The optics for XRR are a $1 / 32^{\circ}$ divergence slit and a Goebel mirror for the incident beam, while a parallel plate collimator and a nickel filter are used for the reflected beam path. Reflectivity data are modeled using the X'Pert reflectivity software package. The crystal structure is determined from x-ray diffractometry (XRD) in Bragg-Brentano geometry using a Goebel mirror for the incident beam. For both XRR and XRD measurements, a copper $\mathrm{K}_{\alpha}$ source (wavelength $0.15418 \mathrm{~nm}$ ) in line focus is used.

Film chemical composition and bonding properties are determined by $\mathrm{x}$-ray photoelectron spectroscopy (XPS). XPS measurements are carried using an AXIS Ultra 
spectrometer in a UHV system (base pressure $\sim 10^{-8} \mathrm{~Pa}$ ), equipped with a

monochromated aluminum $\mathrm{K}_{\alpha} \mathrm{X}$-ray beam, a hemispherical sector analyzer, and a multichannel detector. A $20 \mathrm{eV}$ pass energy resulting in full width at half maximum (FWHM) for the Ag-3d5/2 peak of less than $500 \mathrm{meV}$ is used in order to obtain information from the core-level spectra. Photoelectron spectra are collected as a function of the sample depth using $4 \mathrm{keV} \mathrm{Ar}^{+}$ion-beam etching. Charge-induced shifts of the binding energy are corrected using the $\mathrm{Ar}-2 p$ peak which originates from the $\mathrm{Ar}^{+}$ion sputtering. XPS data is analyzed with Kratos Vision software and the quantitative analysis use the relative sensitivity factors contained within the Vision Software.

\section{RESULTS AND DISCUSSION}

Representative room-temperature resistivities $\rho$ of conductive Ag layers grown in Ar and $\mathrm{Ar} / \mathrm{O}_{2}$ mixtures (for the various $\mathrm{O}_{2}$ deployment schemes described in Section II.A) are plotted as a function of the nominal film thickness $\Theta$ in Fig. 2. For clarity, the plot is divided in two panels. Both panels depict curves that were recorded during film growth in pure $\mathrm{Ar}$ (black squares) and $\mathrm{Ar} / \mathrm{O}_{2}$ mixtures, whereby $\mathrm{O}_{2}$ is present throughout the entire deposition (red stars). Moreover, Fig. 2(a) presents data from experiments in which the growth surface is exposed to $\mathrm{O}_{2}$ for times $t_{E}=2$ and $20 \mathrm{~s}$, and Fig. 2(b) shows $\rho$ vs $\Theta$ curves in which $\mathrm{O}_{2}$ is introduced in the gas ambient after delay times $t_{D}=2$ and 20 s (the corresponding 2 and 20 s curves are represented with green circles and dark yellow diamonds, respectively). All curves show a sharp decrease in $\rho$ with increasing $\Theta$ after which a steady-state resistivity value $\rho^{S S}$ is reached. The nominal film thickness at which 
$\rho^{S S}$ is established marks to the formation of continuous layer (this $\Theta$ value is hereinafter denoted as $\left.\Theta_{\text {cont }}\right)$ as we have previously shown ${ }^{42,53-55}$.

The data in both panels in Fig. 2 show that addition of $\mathrm{O}_{2}$ in the growth atmosphere results in $\Theta_{\text {cont }}$ to decrease from $\approx 81 \mathrm{ML}$ for film grown in pure Ar to $\approx 51$ ML, i.e., the presence of $\mathrm{O}_{2}$ promotes $2 \mathrm{D}$ growth. Furthermore, $\mathrm{Ag}$ growth in an $\mathrm{O}_{2-}$ containing atmosphere yields a steady-state resistivity $\rho^{S S} \approx 1.3 \times 10^{-5} \Omega$-cm, which is larger than the corresponding value of $\approx 1.1 \times 10^{-5} \Omega$-cm for the film grown in pure Ar.

Figure 2(a) reveals that addition of $\mathrm{O}_{2}$ in the gas atmosphere during the early growth stages for a time $t_{E}$ as short as $2 \mathrm{~s}$ is sufficient for decreasing $\Theta_{\text {cont }}$ from $\approx 81 \mathrm{ML}$ (Ar-deposited layer) to $\approx 61 \mathrm{ML}$. By further increasing $t_{E}$ to $20 \mathrm{~s}$, the continuous-layer formation thickness decreases to $\Theta_{\text {cont }} \approx 54$ ML Moreover, $\rho^{S S} \approx 1.1 \times 10^{-5} \Omega$-cm for both $t_{E}$ values presented in Fig. 2(a), which is smaller than the value $\rho^{S S} \approx 1.3 \times 10^{-5} \Omega$-cm for the sample grown under continuous presence of $\mathrm{O}_{2}$, and nearly identical to the resistivity obtained during Ag deposition in pure Ar atmosphere. These trends with respect to both $\Theta_{\text {cont }}$ and $\rho^{S S}$ are qualitatively consistent with our previous observations for the effect of $\mathrm{N}_{2}$ on Ag-layer morphological evolution of ${ }^{42}$, in which we have demonstrated that earlystage-introduction of $\mathrm{N}_{2}$ also promotes $2 \mathrm{D}$ growth without compromising the noblemetal-layer resistivity. Hence, the results in both the present study and in Ref. ${ }^{42}$ underscore that effective and non-invasive surfactant-based growth manipulation strategies can be developed by targeting and selectively modifying early film-formation stages. 


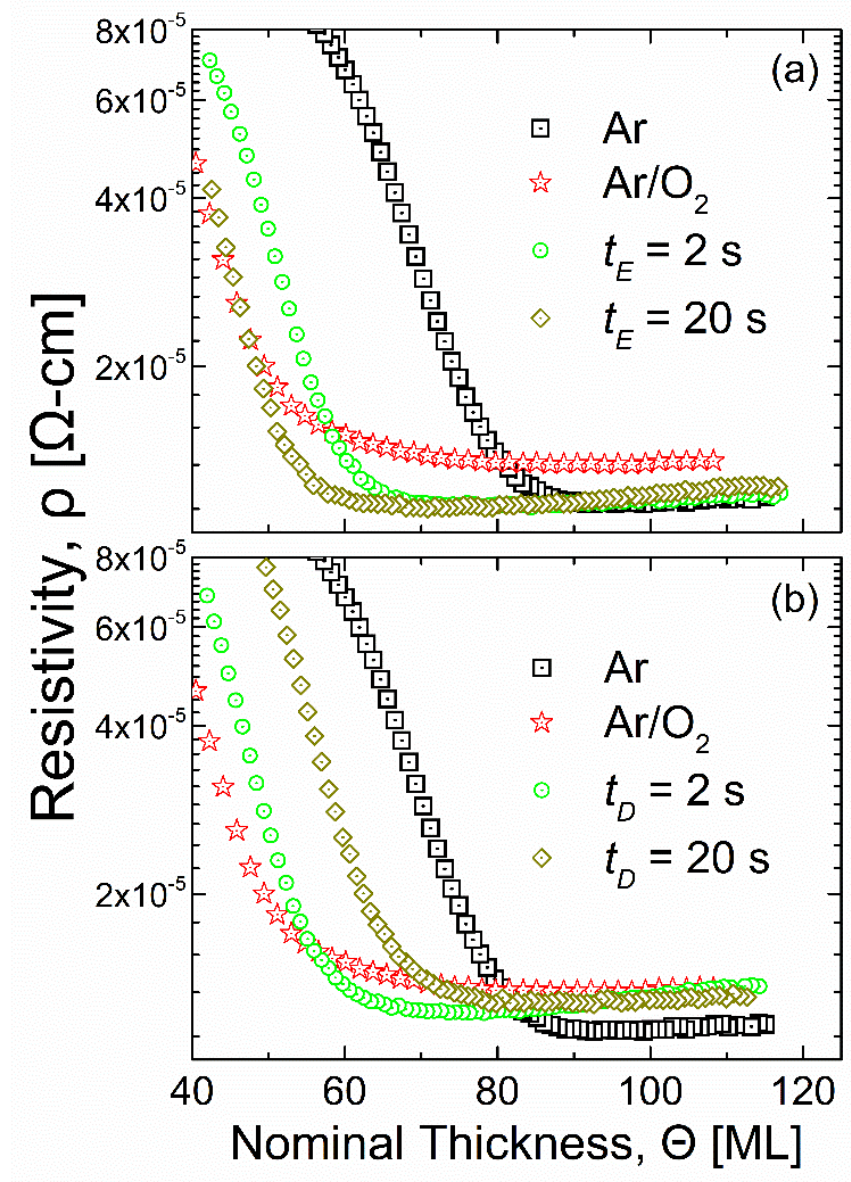

FIG. 2. Resistivity $(\rho) v s$. nominal thickness $(\Theta)$ for: (a) $\mathrm{O}_{2}$ deployment at the early filmgrowth stages for exposure times $t_{E}=2$ and $20 \mathrm{~s}$; (b) $\mathrm{O}_{2}$ deployment at late film-growth stages for delay times $t_{D}=2$ and $20 \mathrm{~s}$. Both panels display curves recorded during growth of samples in pure $\mathrm{Ar}$ and mixed $\mathrm{Ar} / \mathrm{O}_{2}$ ambient, whereby in the latter case the surfactant gas is continuously present until deposition completion.

Delayed $\mathrm{O}_{2}$ deployment (see Fig. 2(b)) with $t_{D}=2$ s yields $\Theta_{\text {cont }} \approx 57 \mathrm{ML}$ which is slightly larger than the value of $\approx 51 \mathrm{ML}$ for the $\mathrm{Ar} / \mathrm{O}_{2}$-grown sample. This value increases further to $\approx 66 \mathrm{ML}$ for $t_{D}=20 \mathrm{~s}$. Concurrently, $\rho^{S S}$ takes values in the range $\sim 1.2 \times 10^{-5}$ to $\sim 1.3 \times 10^{-5} \Omega-\mathrm{cm}$, which is comparable to the steady-state resistivity of the 
$\mathrm{Ar} / \mathrm{O}_{2}$ grown layer, and only slightly higher than the value obtained for films grown in $\mathrm{Ar}$ atmosphere. The trends in Fig. 2(b) are opposite than those found when $\mathrm{N}_{2}$ is used to modify film growth ${ }^{42}$, for which late surfactant gas introduction leads to increase of $\Theta_{\text {cont }}$ and $\rho^{S S}$ above the values for layers grown in pure $\mathrm{Ar}$ atmosphere, i.e., 3D morphological evolution is promoted. This indicates that $\mathrm{O}_{2}$ is more effective in promoting $2 \mathrm{D}$ morphology than $\mathrm{N}_{2}$, since its presence in the gas atmosphere is the only prerequisite for yielding a smaller $\Theta_{\text {cont }}$, relative to films grown in pure Ar.

Multiple studies ${ }^{42,49,53-56}$ have shown that $\rho$ vs. $\Theta$ curves obtained from analysis of in situ spectroscopy ellipsometry data provide a physically correct picture of the morphological evolution of electrically-conductive layers. To confirm that this is the case also for the Ag films in the present study, we perform XRR measurements on a$\mathrm{C} / \mathrm{Ag} / \mathrm{SiO}_{2} / \mathrm{Si}$ stacks, in which continuous Ag layers are grown in $\mathrm{Ar}$ and $\mathrm{Ar} / \mathrm{O}_{2}$ atmospheres. The XRR curves are plotted in Fig.3, whereby circles represent experimental data and solid lines represent the calculated curves, from which the morphology-related quantities of the stack layers are extracted, including the Ag film thickness $h_{f}$, the a-C/Ag interface roughness $w_{a-C / A g}$ (i.e., Ag-layer roughness), and the Ag-layer mass density $\rho_{m}$ (the values for these quantities are also provided in Fig. 3 next to each corresponding reflectivity curve). The analysis shows that $h_{f} \approx 25 \mathrm{~nm}$ for both Ag-layer synthesis conditions, which is in very good agreement with the thicknesses obtained from spectroscopic ellipsometry. Moreover, $\rho_{m}$ is very close to the bulk Ag mass density $\left(10.49 \mathrm{~g} / \mathrm{cm}^{3}\right)^{57}$ as expected for magnetron-sputter-deposited high mobility (i.e., low melting point) metal films ${ }^{58,59}$. We also find that $w_{a-C / A g}=1.8 \mathrm{~nm}$ for the Argrown film, while addition of $\mathrm{O}_{2}$ to the sputtering atmosphere leads to $w_{a-C / A g}=1.5 \mathrm{~nm}$. 
Hence, the results from the XRR analysis confirm that presence of $\mathrm{O}_{2}$ is associated with reduction of roughness at the film growth front, i.e., 2D morphology is promoted.

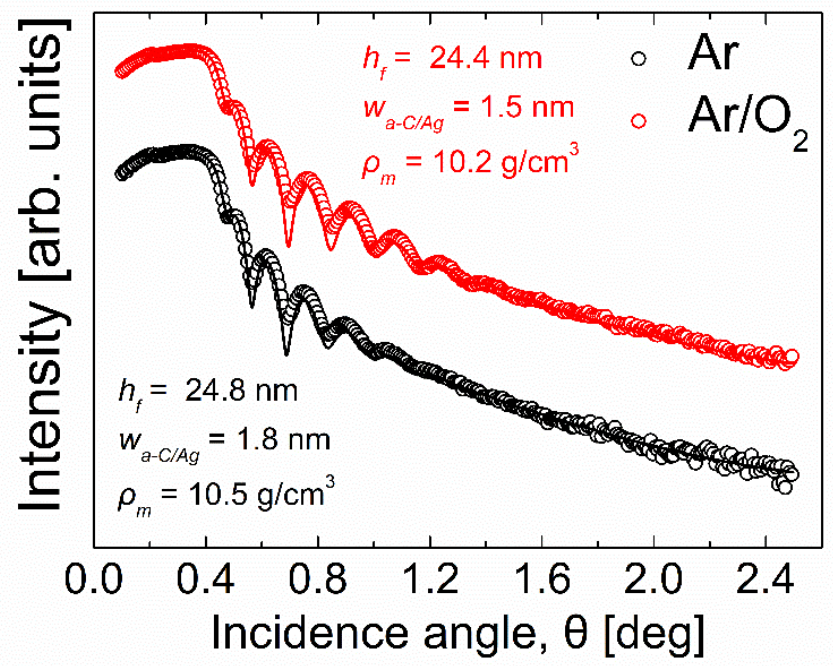

FIG. 3. XRR measurements from a-C/Ag/SiO $2 / S i$ stacks in which $\mathrm{Ag}$ layer is grown in $\mathrm{Ar}$ (black circles) and $\mathrm{Ar} / \mathrm{O}_{2}$-(red circles) atmospheres. The solid lines represent the calculated curves from which the film thickness $h_{f}$, the a-C/Ag interface roughness $w_{a-C / A g}$, and the mass density $\rho_{m}$ are calculated. The values for these quantities for both stacks are also provided in the figure.

In order to establish the effect of the sputtering atmosphere composition on the crystal structure of continuous Ag layers, we investigate the a-C/Ag/SiO $2 / S i$ stacks grown at the conditions reported in Fig. 3 by means of XRD. The corresponding BraggBrentano XRD patterns are shown in Fig. 4 (black and red solid lines for $\mathrm{Ar}-$ and $\mathrm{Ar} / \mathrm{O}_{2}$ grown Ag films, respectively), in which the angular positions of scattering-intensity maxima in unstrained reference $\mathrm{Ag}$ powder and $\mathrm{Si}(100)$ single crystal are marked by vertical dashed lines. The sample grown in Ar atmosphere exhibits a strong 111 
reflection, and a much weaker 200 peak (integrated intensity ratio $\frac{I_{111}}{I_{200}}=8.0$ ), while no other Ag-related diffraction maxima are observed. This indicates that the Ag layer exhibits an [111] out-of-plane texture, as expected for fcc metals. Addition of $\mathrm{O}_{2}$ leads to a more random texture evidenced by the change of the 111 and 200 reflection intensities $\left(\frac{I_{111}}{I_{200}}=3.4\right)$ in the corresponding XRD pattern. Moreover, no diffraction peaks originating from phases other than metallic $\mathrm{Ag}$ (e.g., $\left.\mathrm{AgO}, \mathrm{Ag}_{2} \mathrm{O}\right)$ are detected in Fig. 4.

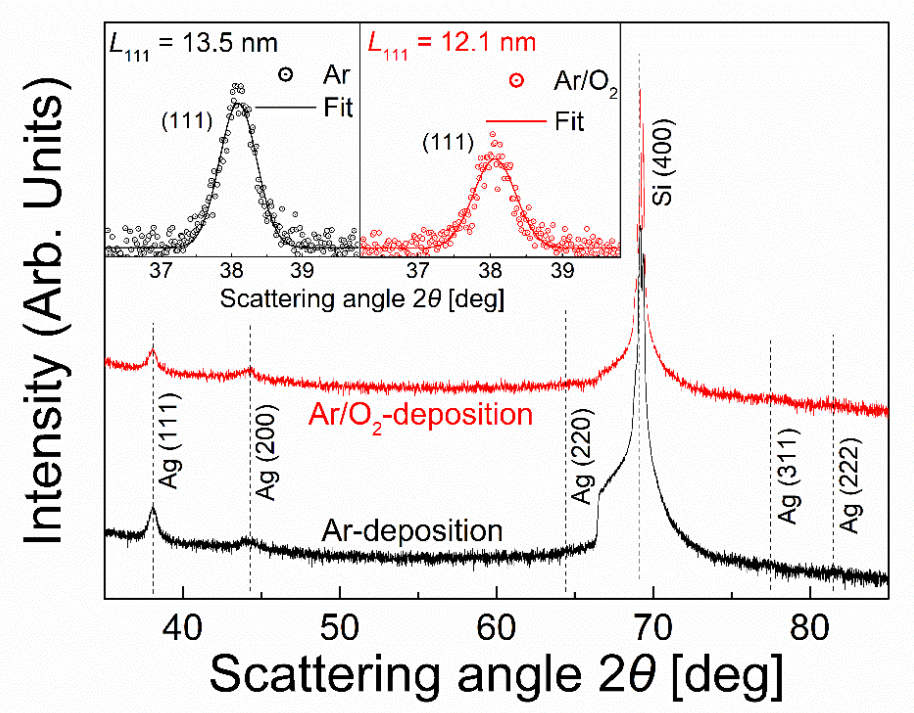

FIG. 4. Bragg-Brentano XRD patterns recorded from a-C/Ag/ $/ \mathrm{SiO}_{2} / \mathrm{Si}$ stacks in which $\sim 25$ nm thick Ag layers are grown in $\mathrm{Ar}$ (black solid line) and $\mathrm{Ar} / \mathrm{O}_{2}$ (red solid line) atmospheres. The vertical dashed lines mark the angular position of XRD reflections in unstrained $\mathrm{Ag}$ powder and $\mathrm{Si}(100)$ crystal. The inset shows a magnified section of the XRD patterns around the $\operatorname{Ag}(111)$ reflections for both samples in which experimental data (circles) are fitted by Gaussian functions (solid lines). From the Gaussian fit the reflection full width at half-maximum is extracted, from which the size of the 111 crystallographic grains $L_{111}$ along the film growth direction is estimated. 
We further analyze the XRD data in Fig. 4 by fitting the 111 reflections with Gaussian functions (see inset in Fig. 4) from which we calculate a full width at halfmaximum (FWHM) of $0.621^{\circ}$ and $0.695^{\circ}$, for $\mathrm{Ar}$ and $\mathrm{Ar} / \mathrm{O}_{2}$-grown samples, respectively. These FWHM values are then used in Scherrer's formula ${ }^{60,61}$ to estimate the size of the 111 crystallites, $L_{111}$, along the growth direction. We find that addition of $\mathrm{O}_{2}$ to the sputtering gas leads to a small decrease of $L_{111}$ from $13.5 \mathrm{~nm}$ to $12.1 \mathrm{~nm}$, i.e., our XRD data indicate that $\mathrm{O}_{2}$ causes grain refinement. The latter can explain the increase of the steady-state resistivity seen in Fig. 2 when $\mathrm{O}_{2}$ is present in the sputtering atmosphere throughout the entire film deposition; decrease of grain size corresponds to increase of the grain boundary area where scattering of charge carriers (electrons) takes place.

The in situ analysis data presented in Fig. 2 indicate that $\mathrm{O}_{2}$ affects the various film-formation stages in a complex fashion. In order to better understand the effect of $\mathrm{O}_{2}$ on the initial growth stages and its correlation with the overall film morphological evolution, we perform SEM analysis on discontinuous layers. Images recorded from a$\mathrm{C} / \mathrm{Ag} / \mathrm{SiO}_{2} / \mathrm{Si}$ stacks, whereby the $\mathrm{Ag}$ layer is grown in $\mathrm{Ar}$ and $\mathrm{Ar} / \mathrm{O}_{2}$ ambient, are shown in Figs. 5 (a) and (b), respectively. For both deposition conditions, data for $\Theta=8,13$, and $21 \mathrm{ML}$ are presented. For $\Theta=8 \mathrm{ML}$, both samples exhibit similar surface topography, featuring nearly-spherical islands with similar size and number density. Increase of $\Theta$ to 13 ML leads to larger islands, but with different shapes depending on the composition of the gas atmosphere; the Ar-grown sample still exhibits nearly-spherical islands, while islands for the $\mathrm{Ar} / \mathrm{O}_{2}$-grown layer become more irregularly shaped. The differences between the two growth conditions become more pronounced for $\Theta=21 \mathrm{ML}$, where 
islands for the sample deposited in $\mathrm{Ar} / \mathrm{O}_{2}$ ambient are more interconnected and elongated, and the substrate areal coverage is larger, as compared to the Ar-grown sample.

The data and trends presented in Figs. 5(a) and (b) are better visualized and quantified by extracting the island size and shape distributions, and calculating the island mean size $(M S)$, island size standard deviation $(S D)$, and the mean in-plane island aspect ratio (AR), for $\Theta=13 \mathrm{ML}$ (Fig. 5(c)) and $\Theta=21 \mathrm{ML}$ (Fig. 5(d)). The results in Fig. 5(c) show similar bell-shaped island size distributions for both conditions with the histogram for $\mathrm{Ar} / \mathrm{O}_{2}$-grown sample (blue bars) being shifted to slightly larger sizes as compared to the corresponding histogram for the Ag layer deposited in pure Ar ambient (red bars). This is also reflected in the calculated $M S \pm S D$ values which are $101.5 \pm 63.3 \mathrm{~nm}^{2}$ (Ar) and $119.2 \pm 71.9 \mathrm{~nm}^{2}\left(\mathrm{Ar} / \mathrm{O}_{2}\right)$. Moreover, the visual impression that the addition of $\mathrm{O}_{2}$ in the gas atmosphere yields more elongated islands is also confirmed by the $A R$ value of 1.75 , relative to $A R=1.47$ for Ar-deposited sample (note that for spherical islands $A R=$ 1). Increase of $\Theta$ to $21 \mathrm{ML}$ (Fig. 5(d)) results in significant differences in the island size distributions, depending on the composition of the gas atmosphere. The sample grown in pure Ar still exhibits a bell-shaped histogram with $M S \pm S D=146.3 \pm 89.8 \mathrm{~nm}^{2}$, while $A R=1.72$. In stark contrast, the island sizes for the $\mathrm{Ar} / \mathrm{O}_{2}$-deposited sample are significantly larger and are distributed over a larger range $(M S \pm S D=591.7 \pm 723.2$ $\left.\mathrm{nm}^{2}\right)$, while the island shapes deviate more clearly from the spherical geometry $(A R=$ 2.07). The overall results in Fig. 5 show that addition of $\mathrm{O}_{2}$ in the gas atmosphere leads to larger and elongated islands for $\Theta>8 \mathrm{ML}$; this behavior has been associated in the literature $e^{42,62-66}$ with incomplete island coalescence. The latter delays cluster reshaping and favors in-plane $v s$. out-of-plane island growth, i.e., a 2D morphology is promoted. 


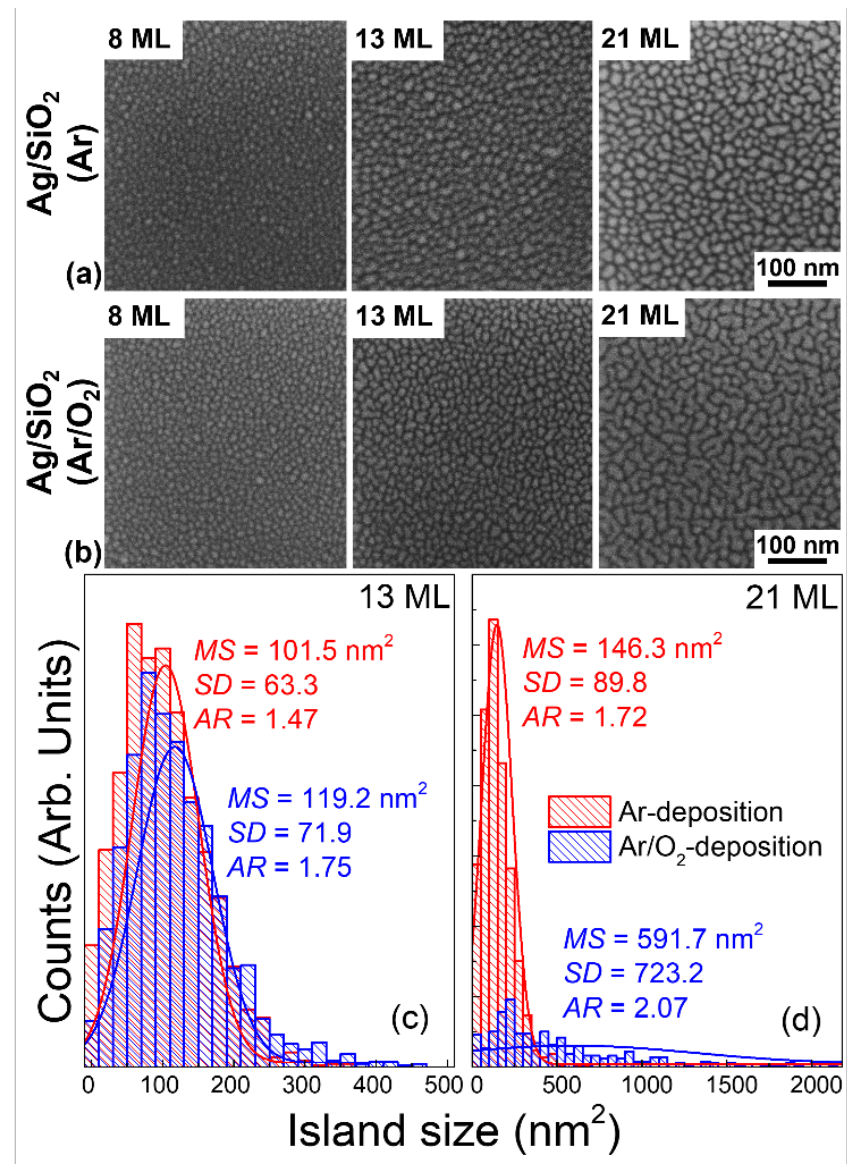

FIG. 5. SEM images of $\mathrm{Ag}$ layers grown in (a) pure $\mathrm{Ar}$, and (b) $\mathrm{Ar} / \mathrm{O}_{2}$ atmospheres for $\Theta$ $=8,13$, and $21 \mathrm{ML}$. Panels (c) and (d) present island size distribution plots and analysis from the data shown in panels (a) and (b), for $\Theta=13$ and $21 \mathrm{ML}$, respectively. The solid blue and red lines in (c) and (d) are generated by fitting the corresponding histogram data to Gaussian functions.

In order to obtain information on the film morphology for $\Theta<8 \mathrm{ML}$ and ascertain whether $\mathrm{O}_{2}$ affects the pre-coalescence film-formation stages of island nucleation and growth, we study the evolution of the optoelectronic properties of discontinuous layers using in situ spectroscopic ellipsometry. Such layers consist of isolated islands and/or 
island clusters and can give rise to $\operatorname{LSPR}^{49}$. We have recently shown ${ }^{42}$ that the LSPRrelated optical response can be effectively modelled by a Lorentz oscillator, while the evolution of the oscillator energy $\hbar \omega_{0}$ as function of nominal thickness $\Theta$ reflects changes in the substrate areal coverage.

Figure 6 plots $\hbar \omega_{0} v s . \Theta$ curves from Ag films in which: (i) the growth surface is exposed to $\mathrm{O}_{2}$ for times $t_{E}=2$ and $20 \mathrm{~s}$ (Fig. 6(a)); and (ii) $\mathrm{O}_{2}$ is introduced in the gas ambient after delay times $t_{D}$ of 2 and $20 \mathrm{~s}$ (Fig. 6(b)). For reference, both panels display curves recorded during growth of samples in pure $\mathrm{Ar}$ (black squares) and mixed $\mathrm{Ar} / \mathrm{O}_{2}$ (red stars) ambient. All curves in Fig. 6 show that the value of $\hbar \omega_{0}$ red-shifts (i.e., decreases) with increasing $\Theta$. This behavior has been attributed to in-plane island growth with continued deposition that leads to decrease of the substrate areal (surface) coverage and the island-island separation distance ${ }^{42,49}$. Moreover, both panels show that addition of $\mathrm{O}_{2}$ to the sputtering atmosphere leads to smaller $\hbar \omega_{0}$ values for a given $\Theta$, while the $\mathrm{Ar} / \mathrm{O}_{2} \hbar \omega_{0} v s . \Theta$ curve exhibits a larger declining slope as compared to its Ar counterpart. This is another indication that presence of $\mathrm{O}_{2}$ promotes in-plane island growth and 2D morphological evolution, and shows that the decrease of $\Theta_{\text {cont }}\left(\right.$ Fig. 2) and $w_{a-C / A g}$ for continuous layers (Fig. 3) has its origin in the initial film-formation stages of island nucleation, growth, and coalescence.

The data in Fig. 6(a) show that, even at a minimum exposure of the growing surface to $\mathrm{O}_{2}$ for $t_{E}=2 \mathrm{~s}$ (which corresponds to $\Theta \approx 1 \mathrm{ML}$ ), the $\hbar \omega_{0} v s$. $\Theta$ slope for $\Theta$ in the range $\approx 2$ to $\approx 5 \mathrm{ML}$ is distinctly steeper than that of the corresponding Ar curve. Earlier simulations of 3D film growth ${ }^{53,54}$ have estimated that the saturation island density — the latter denotes the point during growth until which island nucleation is the 
dominant structure-forming process — is established for $\Theta \approx 0.4 \mathrm{ML}$, when adatoms are the only mobile species on the growing film surface and islands have hemispherical shapes. This value can be further increased when higher-order clusters (i.e., dimers and trimers) are mobile, as, e.g., during growth of high-mobility metals (including Ag) on weakly-interacting substrates ${ }^{59}$. Hence, based on the differences in the slope of the $\hbar \omega_{0}$ vs. $\Theta$ curves for Ar- and $\mathrm{Ar} / \mathrm{O}_{2}$-grown $\left(t_{E}=2 \mathrm{~s}\right)$ films, it cannot be ruled out that $\mathrm{O}_{2}$ promotes 2D growth morphology by: (i) enhancing island densities; and (ii) favoring inplane growth of individual islands through suppression of uphill atomic transport ${ }^{67}$. However, the main mechanism that affects morphology appears to be the decrease of island coalescence completion rate, as seen by the steeper $\hbar \omega_{0} v s . \Theta$ slope at $\Theta>5 \mathrm{ML}$ for the $\mathrm{Ar} / \mathrm{O}_{2}$-deposited $\left(t_{E}=2 \mathrm{~s}\right)$ layer. This notion is also supported by the additional increase of the $\hbar \omega_{0} v s . \Theta$ curve slope when $t_{E}=20 \mathrm{~s}$, which approaches asymptotically that of the $\mathrm{Ar} / \mathrm{O}_{2}$-grown sample. 


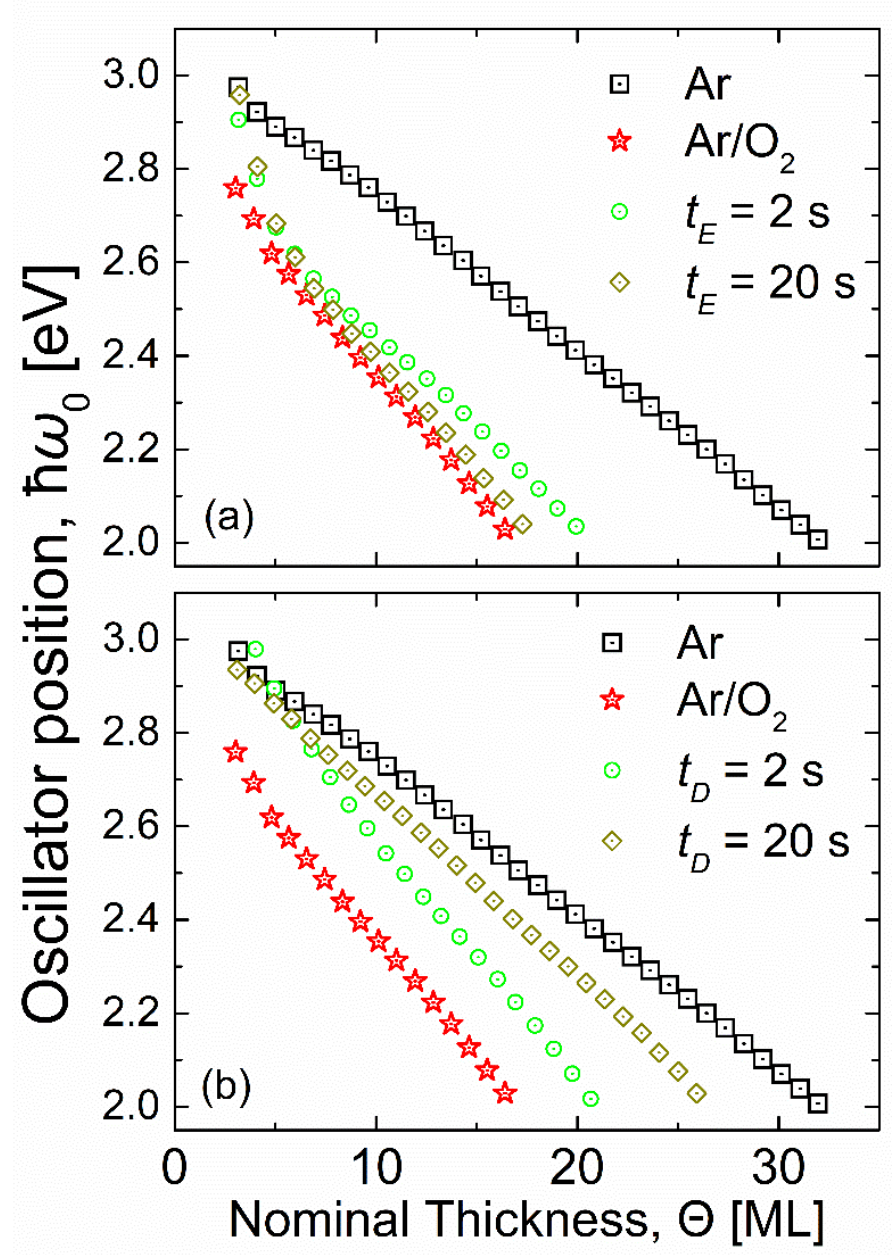

FIG. 6. Lorentz-model resonance energy ( $\left.\hbar \omega_{0}\right)$ vs. nominal thickness $(\Theta)$ for: (a) $\mathrm{O}_{2}$ deployment at the early film-growth stages for exposure times $t_{E}$ between 2 and $20 \mathrm{~s}$; (b) $\mathrm{O}_{2}$ deployment at late film-growth stages for delay times $t_{D}$ between 2 and $20 \mathrm{~s}$. Both panels plot curves recorded during growth of samples in pure $\mathrm{Ar}$ and mixed $\mathrm{Ar} / \mathrm{O}_{2}$ ambient with continuous presence of surfactant gas until deposition completion.

When deposition commences in pure Ar atmosphere and $\mathrm{O}_{2}$ is introduced after a delay time $t_{D}$, Fig. $6(\mathrm{~b})$ shows that the $\hbar \omega_{0} v s$. $\Theta$ curves are nearly identical to that of pure Ar for $\Theta \leq 5 \mathrm{ML}$, above which the curve slope deviates from the Ar line and increases, 
approaching gradually that of the $\mathrm{Ar} / \mathrm{O}_{2}$ data, as $t_{D}$ is decreased from 20 to $2 \mathrm{~s}$. This is consistent with the results in Fig. 2 showing that $\Theta_{\text {cont }}$ increases with increasing $t_{D}$, and again indicates that the effect of $\mathrm{O}_{2}$ on the initial stages of film formation is very crucial for the subsequent overall layer morphology.

To correlate the Ag-layer morphological evolution with changes in the film chemistry and bonding properties, we perform XPS analyses on a-C/Ag/SiO $/ 2$ Si stacks sputter-deposited in $\mathrm{Ar}$ and $\mathrm{Ar} / \mathrm{O}_{2}$ gas atmospheres. Wide XPS scans (not shown here) of both as deposited and $\mathrm{Ar}^{+}$-etched samples exhibit peaks matching the binding energies of all $\mathrm{Ag}$ related electronic orbitals (Ag- $3 s, \mathrm{Ag}-3 p, \mathrm{Ag}-3 d, \mathrm{Ag}-4 s$, and Ag- $4 p$ ), irrespective of the presence or not of $\mathrm{O}_{2}$ in the deposition chamber during $\mathrm{Ag}$ film growth. This is exemplified by the $\mathrm{Ag}-3 d$ high-resolution scans for the $\mathrm{Ar} / \mathrm{O}_{2}$-grown sample presented in Fig. 7. We find that only Ag-Ag bonds form, while no Ag-O peak signature is detected (the expected binding energies for $\mathrm{Ag}_{2} \mathrm{O}$ and $\mathrm{AgO}$ bonds are also marked in Fig. 7). High-resolution scans of the O-1s (Fig. 7 inset) reveal that O-related XPS peaks can be either identified as O-Si bonds ${ }^{68}$ (which emanate from the substrate) or as surface contamination. Moreover, there is no evidence of O-Ag peaks at binding energies 529.0 and $528.5 \mathrm{eV}$, which are correlated to $\mathrm{Ag}_{2} \mathrm{O}$ and $\mathrm{AgO}$ bonds, respectively ${ }^{69,70}$. Hence, the results in Fig. 7 suggest that $\mathrm{O}_{2}$ is only temporarily adsorbed at the film growth front, i.e., it acts as surfactant. However, incorporation of small amounts of oxygen $(<1$ at. $\%$, which is the typical XPS detection limit for light elements ${ }^{71}$ ) cannot be ruled out, most prominently at the film/substrate interface, in accordance with recent literature results $^{42,44,72}$. 


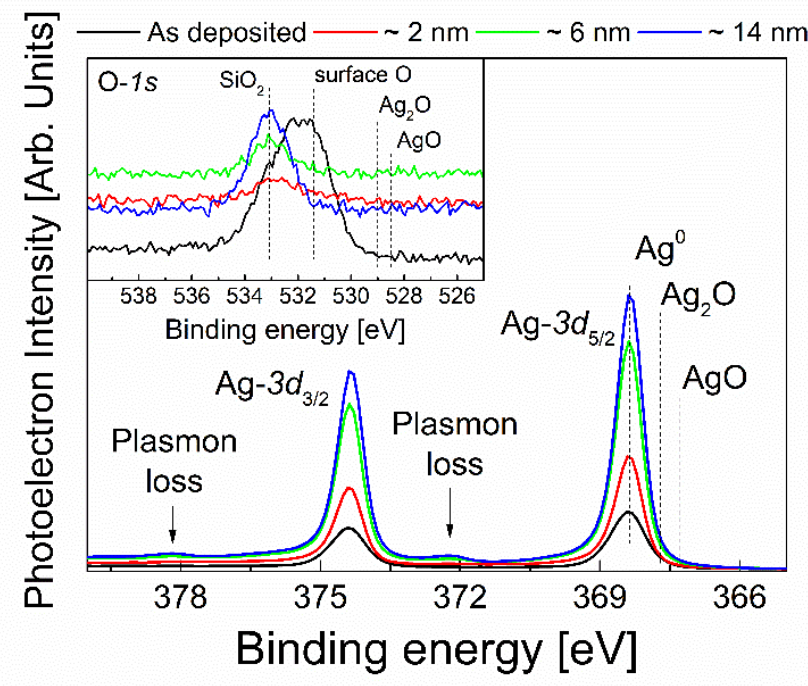

FIG. 7. Ag-3d core-level high-resolution XPS spectra of $\sim 25 \mathrm{~nm}$ thick continuous Ag layers grown by magnetron sputtering on $\mathrm{SiO}_{2} / \mathrm{Si}$ substrates in $\mathrm{Ar} / \mathrm{O}_{2}$ atmospheres. Spectra are recorded from as deposited samples, as well as from samples which have been etched by $\mathrm{Ar}^{+}$ions removing $\sim 2, \sim 6$, and $\sim 14 \mathrm{~nm}$ of material. The inset presents core-level high-resolution scans around the $\mathrm{O}-1 s$ binding energy position. The arrows show the position of Ag plasmon-loss peaks.

In comparison with our previous study on the effect of $\mathrm{N}_{2}$ on Ag-layer morphological evolution ${ }^{42}$, we observe that for the same $\mathrm{Ag}$ vapor deposition rate $\mathrm{a} \sim 10$ lower $\mathrm{O}_{2}$ partial pressure is required for significantly affecting film growth. This can be attributed to the smaller dissociation energy of $\mathrm{O}_{2}$ molecule $(5.2 \mathrm{eV})$ compared to that of $\mathrm{N}_{2}(9.8 \mathrm{eV})^{73}$, which makes generation of reactive atomic species $(\mathrm{O})$ by energetic plasma electrons and incorporation on the growth front more likely. Atomic oxygen has been suggested to favor Ostwald over Smoluchowksi ripening (i.e., cluster diffusion) in the $\mathrm{Ag} / \mathrm{Ag}(100)$ homoepitaxial system ${ }^{72,74,75}$. Concurrently, Ostwald ripening is a much slower process than cluster diffusion, and hence, it is only relevant for post-deposition 
coarsening. From the latter it follows that in an oxygen-free system material will be, primarily, redistributed between islands during growth, while the presence of atomic oxygen will promote material redistribution post-deposition, i.e., coarsening during growth will be hindered. Furthermore, exposure of vicinal Ag surfaces to atomic oxygen has been shown to promote sidewall facet formation ${ }^{76}$, which is known to decrease the rate of material transport between the coalescing islands ${ }^{29,77}$. Moreover, atomic oxygen adsorption on the surface of $\mathrm{Ag}$ islands residing on $\mathrm{SiO}_{2}$ surfaces has been suggested to lower the island surface and the island/substrate interface energies; which yields a smaller driving force for cluster reshaping ${ }^{78}$. The above-mentioned mechanisms are relevant for explaining the hindrance of coalescence completion in our film/substrate system, which is seemingly the process by which 2D growth morphology is promoted for Ag films in the presence of $\mathrm{O}_{2}$.

\section{IV.SUMMARY AND CONCLUSIONS}

The ability to grow noble-metal films with 2D morphologies on weaklyinteracting substrates, including 2D materials and oxides, is essential for the fabrication of high-performance enabling devices. The weak film/substrate interaction provides the driving force toward uncontrolled and pronounced 3D morphological evolution. This tendency can be reversed by using minority less-noble-metal (e.g., transition metals) and gaseous species (both referred to also as surfactants). The mechanisms by which surfactants affect various film growth stages on weakly-interacting substrates are far from being understood, while surfactant-based strategies should be designed in a way that do 
not compromise other physical properties (e.g., optoelectronic properties) of the noblemetal layers.

In the present work, we explore the way by which $\mathrm{O}_{2}$ affects the complex formation stages and the overall morphological evolution of Ag layers deposited by magnetron sputtering on weakly-interacting $\mathrm{SiO}_{2}$ substrates. We combine real-time in situ growth monitoring and ex situ structural and chemical characterization and find that Ag layers grow flatter in the presence of $\mathrm{O}_{2}$, due to incomplete island coalescence. However, $\mathrm{O}_{2}$ causes increase of the Ag-layer electrical resistivity, relative to films grown in pure Ar atmosphere, unless the surfactant gas is only deployed during the initial stages of island nucleation, growth and coalescence. The overall results are consistent with previous findings on the effect of $\mathrm{N}_{2}$ on Ag-layer growth on $\mathrm{SiO}_{2}{ }^{42,43}$; yet significantly smaller amounts of $\mathrm{O}_{2}$ are required to affect morphology owing to its higher reactivity toward Ag compared to that of $\mathrm{N}_{2}$. The knowledge generated herein provides critical insights for the development of non-invasive growth manipulation strategies in which 2D morphology can be promoted by deploying gaseous species, as well as other less-noblemetals, at the film growth with high temporal precision to target and selectively modify critical film formation stages.

\section{ACKNOWLEDGMENTS}

KS acknowledges financial support from Linköping University ("LiU Career Contract, Dnr-LiU-2015-01510, 2015-2020") and the Swedish research council (contract VR-201504630). KS and NP acknowledge financial support from the Olle Engkvist foundation (contract SOEB 190-312) and the Wenner-Gren foundations (contracts UPD2018-0071 
and UPD2019-0007). AJ and GA acknowledge financial support of the French Government program “Investissements d'Avenir" (LABEX INTERACTIFS, reference ANR-11-LABX-0017-01). AJ and KS acknowledge financial support from the Åforsk foundation (contract $\AA F$ 19-137). AD and KS acknowledge financial support through a mobility grant in the framework of the European Consortium of Innovative Universities (ECIU).

${ }^{1}$ T.J. Echtermeyer, S. Milana, U. Sassi, A. Eiden, M. Wu, E. Lidorikis, and A.C. Ferrari, Nano Lett. 16, 8 (2016).

${ }^{2}$ T. Mueller, F. Xia, and P. Avouris, Nat. Photonics 4, 297 (2010).

${ }^{3}$ Y. Xu, C.-Y. Hsieh, L. Wu, and L.K. Ang, J. Phys. D. Appl. Phys. 52, 065101 (2019).

${ }^{4}$ X. Liu, Y. Han, J.W. Evans, A.K. Engstfeld, R.J. Behm, M.C. Tringides, M. Hupalo, H.-Q. Lin, L. Huang, K.-M. Ho, D. Appy, P.A. Thiel, and C.-Z. Wang, Prog. Surf. Sci. 90, 397 (2015).

${ }^{5}$ C. Gong, C. Huang, J. Miller, L. Cheng, Y. Hao, D. Cobden, J. Kim, R.S. Ruoff, R.M. Wallace, K. Cho, X. Xu, and Y.J. Chabal, ACS Nano 7, 11350 (2013).

${ }^{6}$ A. Sclafani and J.-M. Herrmann, J. Photochem. Photobiol. A Chem. 113, 181 (1998).

${ }^{7}$ C.T. Campbell, J. Chem. Soc. Faraday Trans. 92, 1435 (1996).

${ }^{8}$ M. Ohring, The Materials Science of Thin Films (San Diego, 1992).

${ }^{9}$ P.M. Martin, Handbook of Deposition Technologies for Films and Coatings (William Andrew, Amsterdam, 2010).

${ }^{10}$ I. Petrov, P.B. Barna, L. Hultman, and J.E. Greene, J. Vac. Sci. Technol. A Vacuum, 
Surfaces, Film. 21, S117 (2003).

${ }^{11}$ T. Michely and J. Krug, Islands, Mounds and Atoms, 1st ed. (Springer-Verlag Berlin Heidelberg, Berlin, Heidelberg, 2004).

${ }^{12}$ J. Camarero, T. Graf, J.J. de Miguel, R. Miranda, W. Kuch, M. Zharnikov, A.

Dittschar, C.M. Schneider, and J. Kirschner, Phys. Rev. Lett. 76, 4428 (1996).

${ }^{13}$ V. Scheuch, K. Potthast, B. Voigtländer, and H.P. Bonzel, Surf. Sci. 318, 115 (1994).

${ }^{14}$ H. Wolter, M. Schmidt, and K. Wandelt, Surf. Sci. 298, 173 (1993).

${ }^{15}$ D. Kandel and E. Kaxiras, in Solid State Phys., edited by H. Ehrenreich and F.

Spaepen, Vol. 54 (Academic Press, San Diego, 2000), pp. 219-262.

${ }^{16}$ M.H. Hoegen and H. Pietsch, Surf. Sci. 321, L129 (1994).

${ }^{17}$ B. Voigtländer and A. Zinner, Surf. Sci. Lett. 292, L775 (1993).

${ }^{18}$ B. Poelsema, R. Kunkel, N. Nagel, A.F. Becker, G. Rosenfeld, L.K. Verheij, and G. Comsa, Appl. Phys. A Solids Surfaces 53, 369 (1991).

${ }^{19}$ M. Horn-von Hoegen, J. Falta, M. Copel, and R.M. Tromp, Appl. Phys. Lett. 66, 487 (1995).

${ }^{20}$ H.A. van der Vegt, J. Alvarez, X. Torrelles, S. Ferrer, and E. Vlieg, Phys. Rev. B 52, 17443 (1995).

${ }^{21}$ S. Oppo, V. Fiorentini, and M. Scheffler, Phys. Rev. Lett. 71, 2437 (1993).

${ }^{22}$ S. Esch, M. Hohage, T. Michely, and G. Comsa, Phys. Rev. Lett. 72, 518 (1994).

${ }^{23}$ H.A. van der Vegt, H.M. van Pinxteren, M. Lohmeier, E. Vlieg, and J.M.C. Thornton, Phys. Rev. Lett. 68, 3335 (1992).

${ }^{24}$ J. Vrijmoeth, H.A. van der Vegt, J.A. Meyer, E. Vlieg, and R.J. Behm, Phys. Rev. Lett. 72, 3843 (1994). 
${ }^{25}$ J. Camarero, J. Ferrón, V. Cros, L. Gómez, A.L. Vázquez de Parga, J.M. Gallego, J.E. Prieto, J.J. de Miguel, and R. Miranda, Phys. Rev. Lett. 81, 850 (1998).

${ }^{26}$ M. Breeman, G.T. Barkema, M.H. Langelaar, and D.O. Boerma, Thin Solid Films 272, 195 (1996).

${ }^{27}$ B. Lü, G.A. Almyras, V. Gervilla, J.E. Greene, and K. Sarakinos, Phys. Rev. Mater. 2, $063401(2018)$.

${ }^{28}$ V. Gervilla, G.A. Almyras, F. Thunström, J.E. Greene, and K. Sarakinos, Appl. Surf. Sci. 488, 383 (2019).

${ }^{29}$ V. Gervilla, G.A. Almyras, B. Lü, and K. Sarakinos, Sci. Rep. 10, 2031 (2020).

${ }^{30}$ A. Anders, E. Byon, D.-H. Kim, K. Fukuda, and S.H.N. Lim, Solid State Commun. 140, 225 (2006).

${ }^{31}$ D. Gu, C. Zhang, Y.-K. Wu, and L.J. Guo, ACS Nano 8, 10343 (2014).

${ }^{32}$ Logeeswaran VJ, N.P. Kobayashi, M.S. Islam, W. Wu, P. Chaturvedi, N.X. Fang, S.Y. Wang, and R.S. Williams, Nano Lett. 9, 178 (2009).

${ }^{33}$ H. Liu, B. Wang, E.S.P. Leong, P. Yang, Y. Zong, G. Si, J. Teng, and S.A. Maier, ACS Nano 4, 3139 (2010).

${ }^{34}$ C. Furgeaud, L. Simonot, A. Michel, C. Mastail, and G. Abadias, Acta Mater. 159, 286 (2018).

${ }^{35}$ J. Bulír, M. Novotný, A. Lynnykova, J. Lančok, M. Bodnár, and M. Škereň, in Proc. SPIE, edited by R.J. Martin-Palma, Y.-J. Jen, and A. Lakhtakia (2010), p. 77660Q.

${ }^{36}$ G. Zhao, W. Wang, T.-S. Bae, S.-G. Lee, C. Mun, S. Lee, H. Yu, G.-H. Lee, M. Song, and J. Yun, Nat. Commun. 6, 8830 (2015).

${ }^{37}$ W. Wang, M. Song, T.-S. Bae, Y.H. Park, Y.-C. Kang, S.-G. Lee, S.-Y. Kim, D.H. 
Kim, S. Lee, G. Min, G.-H. Lee, J.-W. Kang, and J. Yun, Adv. Funct. Mater. 24, 1551 (2014).

${ }^{38}$ J.M. Riveiro, P.S. Normile, J.P. Andrés, J.A. González, J.A. De Toro, T. Muñoz, and P. Muñiz, Appl. Phys. Lett. 89, 201902 (2006).

${ }^{39}$ A.J. Birnbaum, C. V. Thompson, J.C. Steuben, A.P. Iliopoulos, and J.G. Michopoulos, Appl. Phys. Lett. 111, 163107 (2017).

${ }^{40}$ J. Bulíŕ, M. Novotný, J. Lančok, L. Fekete, J. Drahokoupil, and J. Musil, Surf. Coatings Technol. 228, S86 (2013).

${ }^{41}$ R.H.H. Ko, A. Khalatpour, J.K.D. Clark, and N.P. Kherani, APL Mater. 6, 121112 (2018).

${ }^{42}$ A. Jamnig, N. Pliatsikas, M. Konpan, J. Lu, T. Kehagias, A.N. Kotanidis, N. Kalfagiannis, D. V. Bellas, E. Lidorikis, J. Kovac, G. Abadias, I. Petrov, J.E. Greene, and K. Sarakinos, ACS Appl. Nano Mater. DOI: 10.1021/acsanm.0c00736 (2020).

${ }^{43}$ J. Yun, H.-S. Chung, S.-G. Lee, J.-S. Bae, T.E. Hong, K. Takahashi, S.M. Yu, J. Park, Q. Guo, G.-H. Lee, S.Z. Han, Y. Ikoma, and E.-A. Choi, Nanoscale 12, 1749 (2020).

${ }^{44}$ J.F. Pierson, D. Wiederkehr, and A. Billard, Thin Solid Films 478, 196 (2005).

${ }^{45}$ M. Kawamura, Y. Abe, and K. Sasaki, Thin Solid Films 515, 540 (2006).

${ }^{46}$ R.N. Carter, M.J. Murphy, and A. Hodgson, Surf. Sci. 387, 102 (1997).

${ }^{47}$ C.M. Herzinger, B. Johs, W.A. McGahan, J.A. Woollam, and W. Paulson, J. Appl. Phys. (1998).

${ }^{48}$ C.M. Herzinger, B. Johs, W.A. McGahan, and W. Paulson, Thin Solid Films 313-314, 281 (1998).

49 T.W.H. Oates and A. Mücklich, Nanotechnology 16, 2606 (2005). 
${ }^{50}$ T.W.H. Oates, H. Wormeester, and H. Arwin, Prog. Surf. Sci. 86, 328 (2011).

${ }^{51}$ F. Wooten, Optical Properties of Solids, 1st ed. (Academic Press, New York, 1972).

${ }^{52}$ C.A. Schneider, W.S. Rasband, and K.W. Eliceiri, Nat. Methods 9, 671 (2012).

${ }^{53}$ B. Lü, L. Souqui, V. Elofsson, and K. Sarakinos, Appl. Phys. Lett. 111, 084101 (2017).

${ }^{54}$ B. Lü, V. Elofsson, E.P. Münger, and K. Sarakinos, Appl. Phys. Lett. 105, 163107 (2014).

${ }^{55}$ V. Elofsson, B. Lü, D. Magnfält, E.P. Münger, and K. Sarakinos, J. Appl. Phys. 116, 044302 (2014).

${ }^{56}$ P. Patsalas and S. Logothetidis, J. Appl. Phys. 93, 989 (2003).

${ }^{57}$ A.M. James and M.P. Lord, MacMillan's Chemical and Physical Data, illustrate (Macmillan, London, 1992).

${ }^{58}$ A. Jamnig, N. Pliatsikas, K. Sarakinos, and G. Abadias, J. Appl. Phys. 127, 045302 (2020).

${ }^{59}$ A. Jamnig, D.G. Sangiovanni, G. Abadias, and K. Sarakinos, Sci. Rep. 9, 6640 (2019).

${ }^{60}$ P. Scherrer, in Kolloidchem. Ein Lehrb. (Springer Berlin Heidelberg, Berlin, Heidelberg, 1912), pp. 387-409.

${ }^{61}$ A.L. Patterson, Phys. Rev. 56, 978 (1939).

62 J.M. Warrender and M.J. Aziz, Phys. Rev. B 76, 045414 (2007).

${ }^{63}$ P. Jensen, Rev. Mod. Phys. 71, 1695 (1999).

${ }^{64}$ J. Carrey and J.-L. Maurice, Phys. Rev. B 63, 245408 (2001).

${ }^{65}$ J. Carrey and J.-L. Maurice, Phys. Rev. B 65, 205401 (2002).

${ }^{66}$ B. Lü, E.P. Münger, and K. Sarakinos, J. Appl. Phys. 117, 134304 (2015).

${ }^{67}$ D. McDougall, H. Hattab, M.T. Hershberger, M. Hupalo, M. Horn von Hoegen, P.A. 
Thiel, and M.C. Tringides, Carbon N. Y. 108, 283 (2016).

${ }^{68}$ D.S. Jensen, S.S. Kanyal, N. Madaan, M.A. Vail, A.E. Dadson, M.H. Engelhard, and M.R. Linford, Surf. Sci. Spectra 20, 36 (2013).

${ }^{69}$ J.F. Weaver and G.B. Hoflund, J. Phys. Chem. 98, 8519 (1994).

70 T.C. Kaspar, T. Droubay, S.A. Chambers, and P.S. Bagus, J. Phys. Chem. C 114, $21562(2010)$.

${ }^{71}$ A.G. Shard, Surf. Interface Anal. 46, 175 (2014).

72 A.R. Layson and P.A. Thiel, Surf. Sci. 472, L151 (2001).

${ }^{73}$ Y.-R. Luo, Comprehensive Handbook of Chemical Bond Energies, 1st ed. (CRC Press, Boca Raton, 2007).

${ }^{74}$ A.R. Layson, J.W. Evans, and P.A. Thiel, Phys. Rev. B 65, 193409 (2002).

${ }^{75}$ A.R. Layson, J.W. Evans, V. Fournée, and P.A. Thiel, J. Chem. Phys. 118, 6467 (2003).

${ }^{76}$ J.S. Ozcomert, W.W. Pai, N.C. Bartelt, and J.E. Reutt-Robey, Phys. Rev. Lett. 72, 258 (1994).

${ }^{77}$ N. Combe, P. Jensen, and A. Pimpinelli, Phys. Rev. Lett. 85, 110 (2000).

${ }^{78}$ G. Zhao, E. Jeong, E.-A. Choi, S.M. Yu, J.-S. Bae, S.-G. Lee, S.Z. Han, G.-H. Lee, and J. Yun, Appl. Surf. Sci. 510, 145515 (2020). 